\title{
The boundary element method for the determination of nonlinear boundary conditions in heat conduction
}

\author{
D. Lesnic ${ }^{1}$, T. T. M. Onyango ${ }^{1} \&$ D. B. Ingham ${ }^{2}$ \\ ${ }^{1}$ Department of Applied Mathematics, University of Leeds, UK \\ ${ }^{2}$ Centre for Computational Fluid Dynamics, University of Leeds, UK
}

\begin{abstract}
Physical problems involving heat exchange between the ends of a rod and the surrounding environment can be formulated as a set of equations representing the heat equation and boundary conditions relating the heat fluxes to the difference between the boundary temperatures and the temperature of the surrounding fluid through a function $f$ which represents the heat transfer coefficient. When the heat transfer is purely convective, or solely radiative, then one assumes that $f$ is a linear functional (Newton's law of cooling), or obeys a fourth-order power law (Stefan's law), respectively. However, there are many practical heat transfer situations in which either the governing equation does not take a simple form or the actual method of heat transfer is unknown. In such cases the heat transfer coefficient depends on the boundary temperature and the dependence has a complicated or unknown structure. Processes, such as fast cooling of hot steel or glass in fluids or gases, involve limited opportunities to accurately measure the temperatures and heat fluxes at the surface and in such a case one has to set up an inverse problem that would allows us to reconstruct the exact form of the function $f$. In this study, we investigate a one-dimensional inverse heat conduction problem with unknown nonlinear boundary conditions. We develop the boundary element method to construct and solve numerically the missing terms involving the boundary temperature, the heat flux and the boundary condition law function $f$ which is approximated as a piecewise constant function of temperature. Since the inverse problem under investigation is ill-posed, in order to stabilise the solution we employ the Tikhonov regularization method. Numerical results are presented and discussed.

Keywords: boundary element method, nonlinear boundary conditions, inverse heat conduction problem, Tikhonov's regularization.
\end{abstract}




\section{Introduction}

An interesting, mathematically challenging and well-investigated problem is the identification of coefficients that appear in partial differential equations, e.g. [1,2]. In contrast, the identification of the nonlinear boundary conditions is less welldeveloped. In one-dimensional transient heat conduction these boundary conditions relate the heat flux at the ends of a rod to the boundary temperature through some unknown function $f$. For example, if the heat exchange between the ends of the rod and its environmental surroundings is solely by convection, then one commonly assumes that $f$ is a linear function of the difference in temperature between the ends of the rod and that of the surrounding fluid with the slope given by the heat transfer coefficient (Newton's law of cooling). Identification of a time, space or both space-time dependent heat transfer coefficient in this case has been investigated in, for example [3-5]. For the case of purely radiative transfer of energy, a fourth-power law of the temperature for the function $f$ is usually employed, (Stefan's law), [6].

However, there are many practical heat transfer situations at high temperatures, or in hostile environments, e.g. combustion chambers, cooling steel processes, gas turbines, etc. in which either the actual method of heat transfer is not known, or it cannot be assumed that the governing boundary laws have such a simple form. For example, in the cooling of hot steel or glass in fluids or gases, the heat transfer coefficient depends on the boundary temperature and this dependence has a complicated and unknown structure, [7,8]. From a technical point of view, fast cooling and processes with limited opportunities to accurately measure surface temperatures and/or heat fluxes are of much interest. In such situations one can set up an inverse experiment that would allow the reconstruction (recovery) of the exact form of the function $f$. It is well-known that the identification of nonlinear boundary conditions is an ill-posed problem, [9]. It has been shown elsewhere, [10], that by monitoring (recording, measuring) the transient temperature at one end of the rod then one can recover uniquely the unknown function $f$. However, even if a solution exists and is unique, it will not depend continuously on the input data. Therefore, in order to stabilize the solution one can employ the Tikhonov regularization method, [11], or adopt an engineering approach in which the unknown function $f$ is approximated by a polynomial function with unknown coefficients to be determined.

In this paper we investigate the application of the boundary element method (BEM) for solving numerically the inverse problem of boundary condition law identification in heat conduction.

\section{Mathematical formulation of the inverse problem}

We consider the initial boundary value problem

$$
\frac{\partial T}{\partial t}(x, t)=\frac{\partial^{2} T}{\partial x^{2}}(x, t), \quad(x, t) \in(0,1) \times\left(0, t_{f}\right],
$$




$$
\begin{aligned}
& T(x, 0)=g(x) \quad x \in[0,1], \\
& \frac{\partial T}{\partial n}(0, t)=-\frac{\partial T}{\partial x}(0, t)=f(T(0, t)), \quad t \in\left[0, t_{f}\right], \\
& \frac{\partial T}{\partial n}(1, t)=\frac{\partial T}{\partial x}(1, t)=f(T(1, t)), \quad t \in\left[0, t_{f}\right], \\
& T(0, t)=h(t), \quad t \in\left[0, t_{f}\right],
\end{aligned}
$$

where $T$ represents the unknown temperature of the one-dimensional $\operatorname{rod}(0,1)$, the function $f$ represents the unknown law for the boundary conditions, $t_{f}>0$ is an arbitrary final time of interest, $n$ is the outward unit normal, i.e. $n(0)=-1$, $n(1)=1, g$ is the given initial temperature, $h$ is the given additional measured boundary temperature, and, for simplicity, we have assumed that there are no heat sources. The thermal diffusivity has been taken equal to unity for simplicity. For certain conditions on $f$, the direct problem (2.1)-(2.4) is well-posed, [12]. Moreover, the Fréchet differentiability of the solution $T$ of the direct problem (2.1) (2.4) with respect to $f$ has been established in [13]. The compatibility conditions associated with (2.2)-(2.5) require that $-g^{\prime}(0)=f(g(0)), g^{\prime}(1)=f(g(1))$ and $g(0)=h(0)$. For the inverse problem (2.1)-(2.5) we assume that:

(i) $g \in C^{2+1 / 2}([0,1])$,

(ii) $h \in C^{1+1 / 2}\left(\left[0, t_{f}\right]\right) \quad$ is strictly monotone and $h(0)=g(0)$,

(iii) $T(1, t ; f) \in[h(0), h(t)]$ for all $t \in\left[0, t_{f}\right]$,

where $T(x, t ; f)$ is the solution of the direct mixed problem (2.1), (2.2), (2.4) and (2.5) when $f$ is known. Solvability results for this latter direct problem are given in [14].

It is worth noting that for condition (iii), one cannot guarantee that the range of temperatures on the boundary $x=1$ is contained in the range of temperature measured data (2.5) by giving conditions on the data $g$ and $h$ alone, since condition (iii) depends on the unknown function $f$. However, from the maximum principle for the heat equation, [15], there are easily obtainable conditions under which condition (iii) can be made to hold. For example, if it is known a priori that $f \leq 0$, and if $g(0)=g(1)$, then it is easy to give conditions under which $h$ will be a decreasing function and

$$
h(t)=T(0, t) \leq T(1, t) \leq T(1,0)=g(1)=g(0)=h(0), \quad t \in\left[0, t_{f}\right] .
$$

Under the assumptions (i)-(iii), there is a unique local solution $(T, f)$ of the inverse problem (2.1)-(2.5), [10].

Several factors may allow us to extend this theorem to global solvability. For example, if the admissible (allowable) class of functions $f$ is restricted to uniformly Lipschitz functions $C^{0+1}$, this will be the case, [10]. Other solvability results are given in [16].

At this stage, it is probably difficult to say definitely how ill-posed (unstable) is the inverse problem (2.1)-(2.5), but it may be worth mentioning, [11], that part 
of the ill-posedness comes from the fact that calculating the normal derivative $\frac{\partial T}{\partial n}$ from the boundary Dirichlet noisy data is a typical linear ill-posed problem, $[17,18]$.

\section{The boundary element method (BEM)}

Using the BEM, and applying the initial and boundary conditions (2.2)-(2.4), we obtain the integral representation, e.g. [19],

$$
\begin{aligned}
\eta(x) T(x, t)= & \int_{0}^{t}\left[G(x, t ; \xi, \tau) f(T(\xi, \tau))-T(\xi, \tau) \frac{\partial G}{\partial n(\xi)}(x, t ; \xi, \tau)\right]_{\xi \in\{0,1\}} \mathrm{d} \tau \\
& +\int_{0}^{1} g(y) G(x, t ; y, 0) \mathrm{d} y, \quad(x, t) \in[0,1] \times\left(0, t_{f}\right],
\end{aligned}
$$

where $\eta(0)=\eta(1)=0.5, \eta(x)=1$ for $x \in(0,1)$ and

$$
G(x, t ; \xi, \tau)=\frac{H(t-\tau)}{2 \sqrt{\pi(t-\tau)}} \exp \left[-\frac{(x-\xi)^{2}}{4(t-\tau)}\right]
$$

where $H$ is the Heaviside function.

Applying (3.1) at the boundaries $x=0$ and $x=1$ and using (2.2)-(2.5) we obtain two nonlinear boundary integral equations in the unknowns $f$ and $T(1, t)$, namely

$$
\begin{aligned}
\frac{1}{2} h(t)= & \int_{0}^{t}[G(0, t ; 0, \tau) f(h(\tau))+G(0, t ; 1, \tau) f(T(1, \tau)) \\
& \left.-h(\tau) \frac{\partial G}{\partial n(0)}(0, t ; 0, \tau)-T(1, \tau) \frac{\partial G}{\partial n(1)}(0, t ; 1, \tau)\right] \mathrm{d} \tau \\
& +\int_{0}^{1} g(y) G(0, t ; y, 0) \mathrm{d} y, \quad t \in\left(0, t_{f}\right] \\
\frac{1}{2} T(1, t)= & \int_{0}^{t}[G(1, t ; 0, \tau) f(h(\tau))+G(1, t ; 1, \tau) f(T(1, \tau)) \\
& \left.-h(\tau) \frac{\partial G}{\partial n(0)}(1, t ; 0, \tau)-T(1, \tau) \frac{\partial G}{\partial n(1)}(1, t ; 1, \tau)\right] \mathrm{d} \tau \\
& +\int_{0}^{1} g(y) G(1, t ; y, 0) \mathrm{d} y, \quad t \in\left(0, t_{f}\right] .
\end{aligned}
$$

where

$$
\frac{\partial G}{\partial n(\xi)}(x, t ; \xi, \tau)=\frac{(x-\xi) n(\xi) H(t-\tau)}{4 \sqrt{\pi(t-\tau)^{3}}} \exp \left[-\frac{(x-\xi)^{2}}{4(t-\tau)}\right] .
$$


We discretise the time interval $\left(0, t_{f}\right]$ into a series of $N$ boundary elements, namely

$$
\left(0, t_{f}\right]=\bigcup_{j=1}^{N}\left(t_{j-1}, t_{j}\right]
$$

and assume that the boundary temperature is constant over each boundary element $\left(t_{j-1}, t_{j}\right]$ and takes its value at the mid point $\tilde{t}_{j}=\left(t_{j-1}+t_{j}\right) / 2$, i.e.

$$
T(0, t)=h\left(\tilde{t}_{j}\right)=h_{j}, \quad T(1, t)=T\left(1, \tilde{t}_{j}\right)=T_{1 j}, \quad t \in\left(t_{j-1}, t_{j}\right] .
$$

We also discretise the space interval $[0,1]$ into a series of $N_{0}$ cells, namely

$$
[0,1]=\bigcup_{k=1}^{N_{0}}\left[x_{k-1}, x_{k}\right]
$$

and assume that the initial temperature is constant over each space cell $\left[x_{k-1}, x_{k}\right]$ and takes its value at the mid point $\tilde{x}_{k}=\left(x_{k-1}+k_{k}\right) / 2$, i.e.

$$
T(x, 0)=g\left(\tilde{x}_{k}\right)=g_{k}, \quad \text { for } \quad x \in\left(x_{k-1}, x_{k}\right]
$$

On discretizing the boundary integral equations (3.3) and (3.4), and using the piecewise constant boundary element approximations (3.6) and (3.7), all the BEM integrals that result can be evaluated analytically, [20], and the inverse problem (2.1)-(2.5) recasts as a nonlinear system of $2 N$ equations which, in a generic matrix form, can be written as

$$
A_{f}\left(\mathbf{T}_{1}\right)=\mathbf{b}
$$

where $\mathbf{T}_{1}=\left(T_{1 j}\right)_{j=\overline{1, N}}$, b contains expressions of the known data $g$ and $h$, and $A_{f}$ is a nonlinear operator associated to the unknown function $f$.

\section{Numerical procedure for the inverse problem}

In a previous work, [21], we found the pair solution $(f, T)$ by assuming that $f$ belongs to the global class of polynomial functions with unknown coefficients. In this paper we extend the analysis by assuming that $f$ belongs to a class of piecewise constant functions.

Assuming that the boundary temperature measurement (2.5) is strictly increasing, see condition (ii) of Section 2, we denote by

$$
q_{k}:=h(0)+\frac{k\left(h\left(t_{f}\right)-h(0)\right)}{K}, \quad k=\overline{0, K}
$$

a uniform discretization in $K$ equal sub-intervals of the interval $\left[h(0), h\left(t_{f}\right)\right]$. 
Next we seek a piecewise function $f:\left[q_{0}, q_{K}\right] \rightarrow \mathbb{R}$ defined by

$$
f(T)= \begin{cases}a_{1}, & T \in\left[q_{0}, q_{1}\right), \\ a_{2}, & T \in\left[q_{1}, q_{2}\right), \\ \vdots & \\ a_{K}, & T \in\left[q_{K-1}, q_{K}\right],\end{cases}
$$

where the coefficients $\mathbf{a}=\left(a_{k}\right)_{k=\overline{1, K}}$ are unknown and yet to be determined by imposing (4.3) in a nonlinear least-squares sense. From (4.1) and the assumption that the condition (iii) of Section 2 is satisfied we have

$$
f\left(h\left(\widetilde{t_{l}}\right)\right)=a_{\varphi(l)}, f\left(T\left(1, \tilde{t}_{l}\right)\right)=a_{\psi(l)}, \quad l=\overline{1, N},
$$

where for each $l \in\{1, \ldots, N\}, \varphi(l)$ is the unique number in the set $\{1, \ldots, K\}$ such that $h\left(\tilde{t}_{l}\right) \in\left[q_{\varphi(l)-1}, q_{\varphi(l)}\right)$, and $\psi(l)$ is the unique number in the set $\{1, \ldots, K\}$ such that $T\left(1, \tilde{t}_{l}\right) \in\left[q_{\psi(l)-1}, q_{\psi(l)}\right)$. Based on (4.2), the system of nonlinear equations (3.8) is solved using the minimization of the Tikhonov regularization functional $S: \mathbb{R}^{K} \times \mathbb{R}^{N} \rightarrow \mathbb{R}_{+}$defined by

$$
S\left(\mathbf{a}, \mathbf{T}_{\mathbf{1}}\right)=\left\|A_{f}\left(\mathbf{T}_{1}\right)-\mathbf{b}\right\|^{2}+\kappa\|a\|^{2},
$$

where $\kappa \geq 0$ is the regularization parameter to be prescribed.

In (4.3) we have included the regularization term in order to stabilize the solution of the inverse and ill-posed problem under investigation with respect to noisy errors in the input measurements (2.5), namely

$$
h^{\epsilon}(t)=h(t)+\epsilon,
$$

where $\epsilon$ are Gaussian random variables with mean zero and deviation $\sigma=\rho \times$ $\max \left\{|h(t)|, t \in\left[0, t_{f}\right]\right\}$ generated using the NAG routine G05DDF, and $100 \rho$ represents the percentage of noise. The minimization of the functional (4.3) is performed using the NAG routine E04FCF. This routine is minimizing iteratively an arbitrary sum of squares with no constraints on the variables and no gradient needed to be supplied by the user.

\section{Numerical results and discussion}

In this section we illustrate and discuss numerical results for two benchmark test examples consisting of retrieving the pair solution $(f, T)$ satisfying (2.1)-(2.5), when $f$ has the piecewise constant parametrization (4.1). Higher-order (e.g linear, quadratic) piecewise constant polynomial approximations are deferred to a future work.

The BEM described in Section 3 is applied with $\left(N, N_{0}\right)=(40,40)$ to generate the forward nonlinear operator $A_{f}\left(T_{1}\right)$. The parametrization (4.1) is sought with a typical value of $K=10$ large enough to capture all the significant features of the examples below. 
The initial guess for minimizing the functional (4.3) was taken $\left(a^{0}, T_{1}^{0}\right)=(3,3)$ for Example 1 and $\left(a^{0}, T_{1}^{0}\right)=(3,50)$ for Example 2 . In both examples, the analytical temperature function to be retrieved was taken to be

$$
T(x, t)=x^{2}-x+1+2 t, \quad(x, t) \in[0,1] \times\left[0, t_{f}\right] .
$$

Equation (5.1) then generates the initial condition (2.2) and the measurement boundary condition (2.5) be given by

$$
T(x, 0)=g(x)=x^{2}-x+1, \quad x \in[0,1],
$$

and

$$
T(0, t)=h(t)=1+2 t, \quad t \in[0,1] .
$$

Remark that for the example (5.1) conditions (i)-(iii) of Section 2 are satisfied such that the local solvability of the inverse problem (2.1)-(2.5) is ensured.

\subsection{Example 1}

In this simple example, we solve for $T$ and $f$ the inverse problem in the domain $(0,1) \times\left(0, t_{f}=1\right]$ given by the heat equation (2.1) subject to the initial condition (5.2), the boundary conditions (2.3) and (2.4), and the additional measurement (5.3). The analytical solution to be retrieved is given by equation (5.1) for the temperature $T(x, t)$ and $f(T) \equiv 1$, i.e. the constant vector $\mathbf{a} \equiv \mathbf{1}$.

Figures 1(b) and (c) show the numerical results for $\mathbf{T}_{\mathbf{1}}$ and $\mathbf{a}$, respectively, when the input measured data (5.3) is contaminated by various amounts of additive noise $\rho \%$, as in (4.4), see Figure 1(a). Whilst from Figure 1(b) it can be seen that the analytical value of $T(1, t)=1+2 t$ is excellently retrieved almost independent of $\rho$, from Figure 1(c) it can be seen that the numerically obtained solution for $\mathbf{a}$ is proportionally accurate with the amount of noise $\rho$ introduced in the input data. This indicates that we have obtained a stable solution. We also report that no regularization, i.e. $\kappa=0$ in (4.3), was found necessary for this simple example.

\subsection{Example 2}

It is well-known that, in general, the heat flux on a surface is the sum of two terms corresponding to convection and surface radiation, [6]. We therefore examine an interesting and important example of a nonlinear inverse heat conduction problem in which the relation between the heat flux and temperature at the boundaries, from a physical point of view, is a fourth power in the temperature and this represents radiative boundary conditions, i.e. we seek to retrieve $f(T)=T^{4}$. In this case, the boundary conditions (2.3) and (2.4) are slightly modified as

$$
\frac{\partial T}{\partial n}(x, t)=f(T(x, t))+1-(1+2 t)^{4}, \quad(x, t) \in\{0,1\} \times\left(0, t_{f}\right],
$$

such that the temperature (5.1) and the nonlinear boundary law $f(T)=T^{4}$ represent the analytical solution of the inverse problem (2.1), (5.2)-(5.4). 


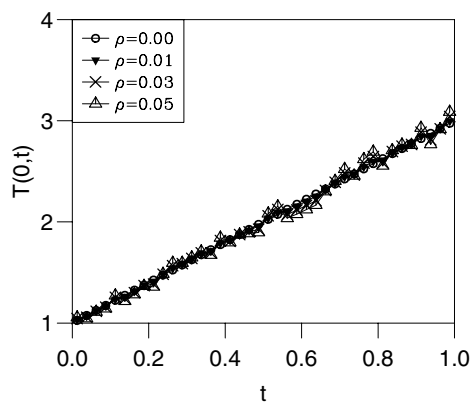

(a)

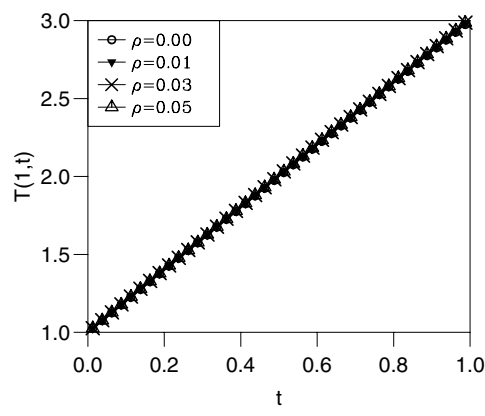

(b)

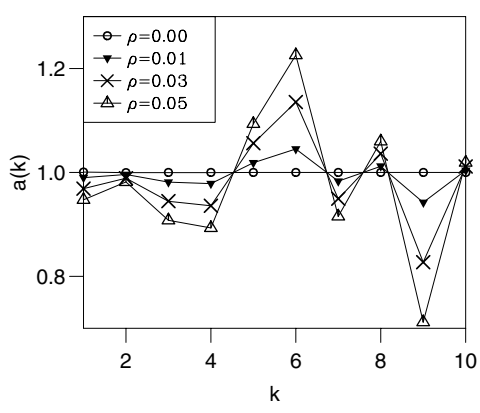

(c)

Figure 1: (a) The analytical boundary temperature $T(0, t)$, (b) the numerical boundary temperature $T(1, t)$, as functions of time $t$, and (c) the numerical vector $\mathbf{a}=\left(a_{k}\right)_{k=\overline{1,10}}$, when the amount of noise in (4.4) is: (o) $\rho=0,(\boldsymbol{\nabla}) \rho=0.01,(\times) \rho=0.03,(\triangle) \rho=0.05$. No regularization parameter, $\kappa=0$.

Figures 2(a) and (b) show the results for the boundary temperature $T(1, t)$ and the function $f(T)$, respectively, when various amounts of noise $\rho \in\{0,1,3,5\} \%$ are included in the measured data (5.3), as shown in Figure 1(a). No regularization has been added in the functional (4.3). From Figure 2 it can be seen that as the amount of noise $\rho$ decreases the numerical solution approaches the exact solution. The numerical results can be further improved by employing some regularization, say $\kappa=10^{-3}$, in (4.3), as shown in Figure 3.

\section{Conclusions}

In this paper, we have investigated an inverse heat conduction problem with unknown nonlinear boundary conditions. We have used the BEM in conjunction with the Tikhonov regularization procedure to construct and solve numerically the 


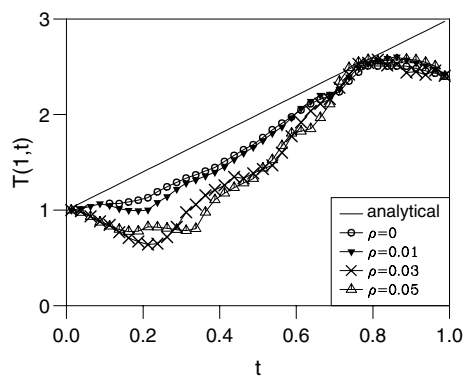

(a)

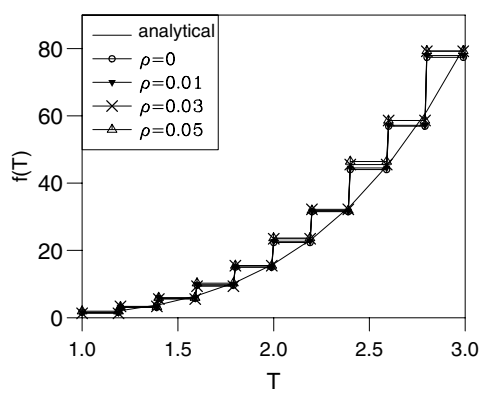

(b)

Figure 2: The analytical and numerical approximations of (a) the boundary temperature $T(1, t)$ and (b) the function $f(T)$, when $\rho \in\{0,1,3,5\} \%$ and $\kappa=0$.

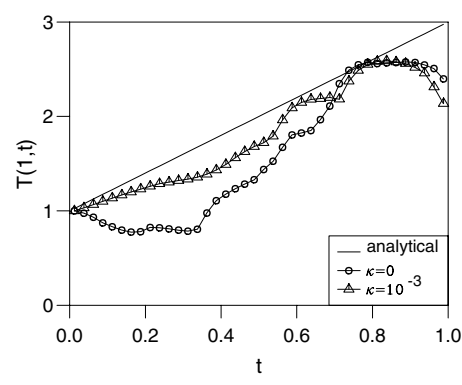

(a)

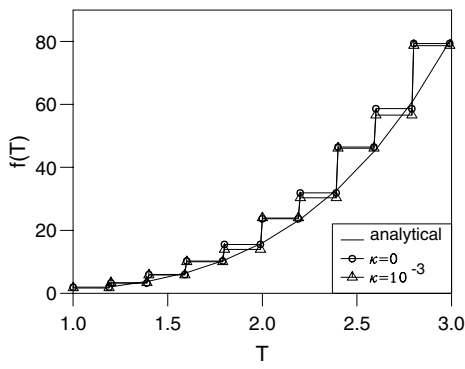

(b)

Figure 3: The analytical and numerical approximations of (a) the boundary temperature $T(1, t)$ and (b) the function $f(T)$, when $\rho=5 \%, \kappa=0$ and $10^{-3}$.

missing terms involving the boundary temperature, the heat flux, and a piecewise constant approximation of the function relating the boundary temperature and heat flux in one-dimensional transient heat conduction. The numerical results obtained showed that a stable and accurate solution was obtained. Future work will concern extensions to higher dimensions.

\section{References}

[1] N.V. Muzylev, Uniqueness theorems for some converse problems of heat conduction, U.S.S.R. Comput. Maths. Math. Phys., 20, 120-134, (1980).

[2] M. Pilant and W. Rundell, Undetermined coefficient problems for nonlinear elliptic and parabolic equations, Int. Ser. Numer. Math., 77, 139-154, (1986). 
[3] S. Chantasiriwan, Inverse heat conduction problem of determining timedependent heat transfer coefficients, Int.J. Heat Mass Transfer, 42, 42754285, (1999).

[4] E. Divo, A.J. Kassab, J.S. Kapat and M.-K Chyu, Retrieval of multidimensional heat transfer coefficient distributions using an inverse BEM-based regularized algorithm: numerical and experimental results, Eng. Anal. Boundary Elements, 29, 150-160, (2005).

[5] C.-H. Huang, D.-M. Wang and H.-M. Chen, Prediction of local thermal contact conductance in plate finned-tube heat exchangers, Inverse Problems in Engineering, 7, 119-141, (1999).

[6] H.S. Carslaw and J.C. Jaeger, Conduction of Heat in Solids, 2nd edition, Clarendon Press, Oxford (1959).

[7] A. Rösch, A Gauss-Newton method for the identification of non-linear heat transfer laws, Int. Ser. Numer. Math., 139, 217-230, (2002).

[8] D.H. Wolf, F.P. Incropera and R. Viskanta, Jet impingement boiling, $A d v$. Heat Transfer, 23, 1-132, (1993).

[9] A. Rösch, Identification of nonlinear heat transfer laws by optimal control, Numer. Funct. Anal. Optimiz., 15, 417-434, (1994).

[10] M. Pilant and W. Rundell, An iteration method for the determination of an unknown boundary condition in a parabolic initial-boundary value problem, Proc. Edinburgh Math. Soc., 32, 59-71, (1989).

[11] T. Kaiser and F. Tröltzsch, An inverse problem arising in the steel cooling process, Wissenschaftliche Zeitung TU Karl-Marx-Stadt, 29, 212-218, (1987).

[12] A. Rösch, Identification of nonlinear heat transfer laws by means of boundary data, In Progress in Industry, Wiley, Teubner, 405-412, (1996).

[13] A. Rösch, Fréchet differentiability of differential of the solution of the heat equation with respect to a nonlinear boundary condition, Z. Anal. Anw. 15, 603-618, (1996).

[14] A. Friedman, Partial Differential Equations of Parabolic Type, Prentice Hall, Englewood Cliffs, N.J. (1964).

[15] M.H. Protter and H.F. Weinberger, Maximum Principles in Differential Equations, Prentice-Hall, Englewood Cliffs, N.J. (1967).

[16] A. Shidfar and H. R. Nikoofar, An inverse problem for a linear diffusion equation with non-linear boundary conditions, Appl. Math. Lett., 2, 385-388, (1989).

[17] W. Gerlach and L. von Worfersolorf, On approximate computation of the values of the normal derivative of solutions to linear partial differential equations of second order with applications to Abel's integral equation, ZAMM, 66, 31-36, (1986).

[18] R. Bialecki, E. Divo and A.J. Kassab, Reconstruction of time-dependent boundary heat flux by a BEM based inverse algorithm, Eng. Anal. Boundary Elements, 30, 767-773, (2006).

[19] C.A. Brebbia, J.C.F. Telles and L.C. Wrobel, Boundary Element Techniques, Springer-Verlag, Berlin (1984). 
[20] T.T.M Onyango, D.B. Ingham and D. Lesnic, Restoring boundary conditions in heat conduction, J. Eng. Math., 62, 85-101, (2008).

[21] T.T.M. Onyango, D.B. Ingham and D. Lesnic, Reconstruction of boundary condition laws in heat conduction using the boundary element method, Comput. Math. Appl., 57, 157-168, (2009). 